\title{
Synthesis and Properties of MPEG-Coated Superparamagnetic Magnetite Nanoparticles
}

\author{
Xueli Cao, ${ }^{1}$ Baolin Zhang, ${ }^{2}$ Fangyuan Zhao, $^{2}$ and Lingyun Feng ${ }^{2}$ \\ ${ }^{1}$ College of Science, Guilin University of Technology, Guilin 541004, China \\ ${ }^{2}$ State Key Laboratory Breeding Base of Nonferrous Metals and Specific Materials Processing, \\ School of Materials Science and Engineering, Guilin University of Technology, Guilin 541004, China
}

Correspondence should be addressed to Baolin Zhang, baolinzhang@ymail.com

Received 29 July 2012; Accepted 24 August 2012

Academic Editor: Mao-Wang Lu

Copyright (C) 2012 Xueli Cao et al. This is an open access article distributed under the Creative Commons Attribution License, which permits unrestricted use, distribution, and reproduction in any medium, provided the original work is properly cited.

\begin{abstract}
The magnetite nanoparticles were synthesized by the thermal decomposition of iron(III) acetylacetonate in methoxy polyethylene glycol, which was used as solvent, reducing agent, and modifying agent in the reaction. The morphologies and phase compositions of the nanoparticles were determined by transmission electron microscopy and X-ray diffraction, respectively. The surface coating of the nanoparticles was recognized using Fourier transform infrared spectroscopy. Magnetic properties were measured using superconducting quantum interference device. The zeta potential and hydrodynamic size of the nanoparticles was determined using nanoparticle and zeta potential analyzer. The magnetite nanoparticles show superparamagnetic behavior in $300 \mathrm{~K}$. The negatively charged methoxy polyethylene glycol-coated magnetite nanoparticles in water exhibited longer-time dispersion with small hydrodynamic size than the magnetite nanoparticles synthesized by the thermal decomposition of iron(III) acetylacetonate in polyethylene glycol. The less conjunction between methoxy polyethylene glycol-coated magnetite nanoparticles due to the inert $-\mathrm{CH}_{3}$ terminal group may cause their higher stability in water dispersion.
\end{abstract}

\section{Introduction}

Magnetite $\left(\mathrm{Fe}_{3} \mathrm{O}_{4}\right)$ nanoparticles (MNPs) with appropriate surface modifications are promising as contrast agents with long blood half-life in magnetic resonance imaging (MRI) such as MR angiography, or cerebral blood pool imaging for fMRI (functional MRI) [1-14]. Monodisperse MNPs with high crystallinity and narrow size distribution have been synthesized by high-temperature organic phase decomposition methods [15-19]. However, those magnetite nanocrystals are only well dispersible in nonpolar solvents because they have been capped by hydrophobic surfactant ligands, which limits their applications in the biomedical field unless a further surface modification is employed. PEG (polyethylene glycol) or MPEG (methoxy polyethylene glycol)-coated MNPs as useful contrast agents show a long blood circulation time because of the protein rejecting ability of PEG [3, 6, 8, 20-22], but their preparation processes reported in the literatures are complex. MNPs with small sizes and good magnetization were synthesized by the polyol process $[23,24]$. Because the aggregations of the MNPs in water take place with time due to the interaction of the nanoparticles, extra treatments have to be exploited to prevent the aggregation $[25,26]$. In this work, we synthesized the MNPs in a simple way by the thermal decomposition of iron(III) acetylacetonate $\left(\mathrm{Fe}(\mathrm{acac})_{3}\right)$ in MPEG and investigated their properties.

\section{Experimental}

All the chemicals were analytical purity and used without further purification. MNPs were synthesized by the thermal decomposition of $\mathrm{Fe}(\mathrm{acac})_{3}$ in MPEG (MPEG-1200, $\mathrm{Mw}=$ $1200,99 \%)$ at elevated temperature. In general, $2 \mathrm{mmol}$ of $\mathrm{Fe}(\mathrm{acac})_{3}(98 \%)$ was dissolved in $15 \mathrm{~mL}$ MPEG-1200 during magnetically stirring under a flow of argon. The solution was dehydrated at $120^{\circ} \mathrm{C}$ for $1 \mathrm{~h}$, then quickly heated to $200^{\circ} \mathrm{C}$ and kept at this temperature for $30 \mathrm{~min}$. Then the temperature was elevated to $320^{\circ} \mathrm{C}$ and kept for another $30 \mathrm{~min}$. The black solutions were cooled at room temperature and then 


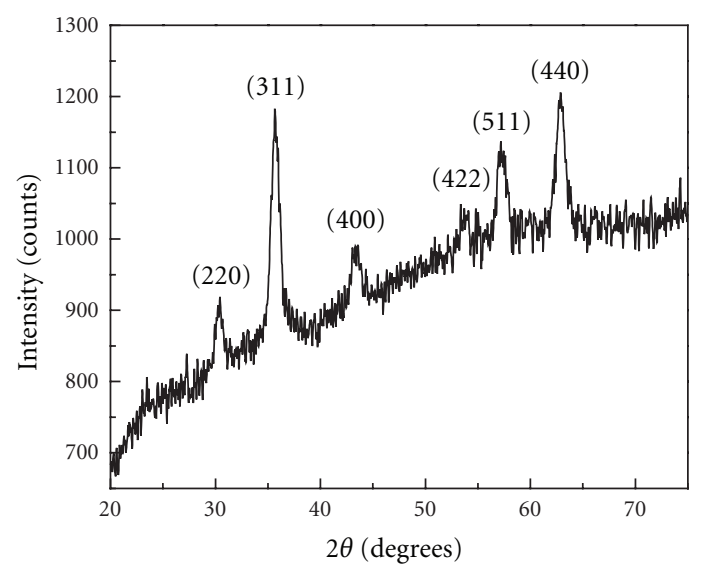

FIGURE 1: XRD patterns of the magnetite nanoparticles synthesized by the thermal decomposition of Fe(acac) $)_{3}$ in MPEG-1200.

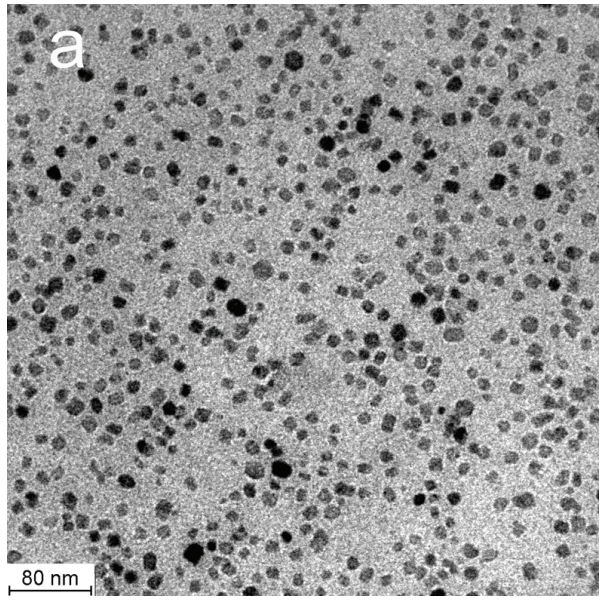

(a)

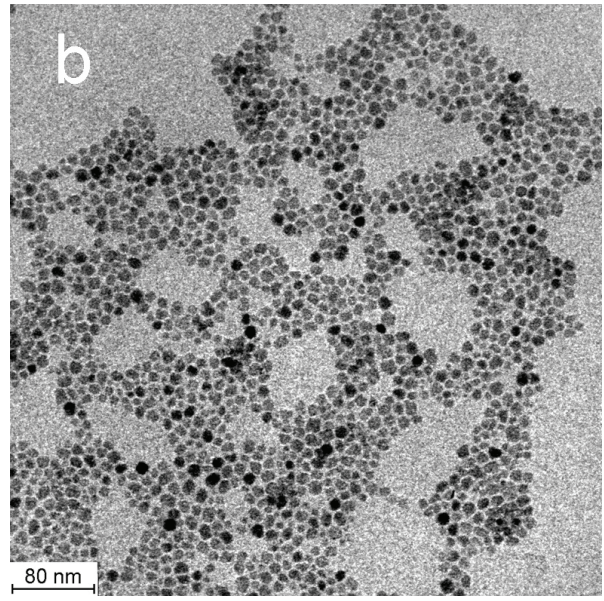

(b)

FIGURE 2: TEM images of the magnetite nanoparticles synthesized by the thermal decomposition of Fe(acac) 3 in (a) MPEG-1200; (b) PEG1000.

were mixed with $60 \mathrm{~mL}$ of toluene. After ultrasonic stirring, the particles were precipitated by the adsorption of magnet and washed two times with acetone to remove the excess organics. The black powders were obtained by drying the precipitates in vacuum and then dispersed in water. For comparison, MNPs were also synthesized by the thermal decomposition of $\mathrm{Fe}(\mathrm{acac})_{3}$ in PEG (PEG-1000, $\mathrm{Mw}=1000$, 99\%) according to the procedure introduced in the literature [24].

Size and morphology of the nanoparticles were determined by transmission electron microscopy (TEM, JEOL2010) and the crystal structure was identified using Xray diffraction (XRD, X'Pert PRO, PANalytical). Surface coating and surface charge of the nanoparticles were recognized using Fourier transform infrared spectroscopy (FTIR, Nicolet Nexus 470, Thermo Electron) and zeta potential measurements (Nano ZS900, Malvern). Magnetic properties were determined using superconducting quantum interference device (SQUID, MPMS XL-7, Quantum Design) measurements.

\section{Results and Discussion}

Figure 1 shows the XRD pattern of the particles synthesized by the thermal decomposition of $\mathrm{Fe}(\mathrm{acac})_{3}$ in MPEG-1200, which matches well with the standard XRD patterns of bulk magnetite (JCPDS File no. 01-085-1436) indicating that the particles contain $\mathrm{Fe}_{3} \mathrm{O}_{4}$ phase. The peaks with $2 \theta$ values of $30.09^{\circ}, 35.44^{\circ}, 43.07^{\circ}, 53.43^{\circ}, 56.96^{\circ}$, and $62.54^{\circ}$ correspond to the crystal planes (220), (311), (400), (422), (511), and (440) of crystalline $\mathrm{Fe}_{3} \mathrm{O}_{4}$, respectively. The widths of these peaks obviously are broaden and the average crystallite size calculated using Scherrer's equation gives $9.3 \mathrm{~nm}$ for (311) peak, which is consistent with the size measured in the TEM image (Figure 2(a)), indicating the single crystal nature of the particles. Figure 2 shows the TEM morphologies of MNPs synthesized in MPEG and in PEG, TEM samples were made by dropping the water dispersion onto carbon-coated copper grids and drying them under room temperature. The average sizes of the particle are $9.5 \pm 1.7 \mathrm{~nm}$ and $10.1 \pm 2 \mathrm{~nm}$ for MPEG-1200 and PEG-1000 as reactants, respectively. 


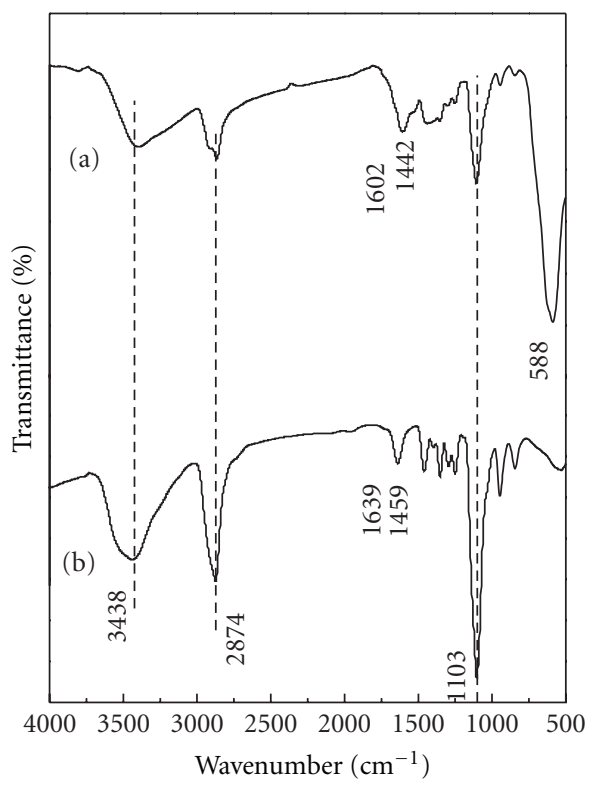

FIGURE 3: FT-IR spectra of (a) the magnetite nanoparticles coated with MPEG-1200; (b) MPEG-1200.

Figure 3 shows the FTIR spectra for the MNPs synthesized in MPEG-1200 and pure MPEG-1200. New peaks can be found at about $1602 \mathrm{~cm}^{-1}, 1442 \mathrm{~cm}^{-1}$, and $588 \mathrm{~cm}^{-1}$ by comparing curve (a) and curve (b). The peaks at $1602 \mathrm{~cm}^{-1}$, $1442 \mathrm{~cm}^{-1}$ can be assigned to the $\mathrm{COO}^{-}$vibrations [23, 24], the strong absorption band at about $580 \mathrm{~cm}^{-1}$ is due to $\mathrm{Fe}-$ $\mathrm{O}$ stretching vibration for the $\mathrm{Fe}_{3} \mathrm{O}_{4}$ nanoparticles $[23,24]$. This suggests that $-\mathrm{CH}_{2}-\mathrm{OH}$ group has been oxidized to$\mathrm{COO}^{-}$group during the reaction. The reaction at the $-\mathrm{OH}$ sites [24], the subsequently $\mathrm{Fe}_{3} \mathrm{O}_{4}$ nucleation, and grain growth could be the mechanism of the formation of $\mathrm{Fe}_{3} \mathrm{O}_{4}$ nanoparticles.

Figure 4 shows the zeta potential measurement of the MPEG-coated MNPs dissolved in deionized water. The zeta potential is about $-21 \mathrm{mV}$ that provides electrostatic repulsion forces between nanoparticles. Figure 5 shows the images of the MNPs modified by MPEG-1200 and by PEG-1000 in water with the concentration at about $5 \mathrm{mg} / \mathrm{mL}$. the MPEGcoated MNPs had been dispersed well in water for more than 3 months and no flocculates or precipitates were observed. However, the MNPs modified by PEG started to precipitate in water for several days, which surely would impair their application. It did not produce much difference to the results when we tried to wash the nanoparticles with acetone or ethyl acetate according to the experiment procedure or to vary the synthesis temperature between $240-320^{\circ} \mathrm{C}$. Therefore, although synthesized in and modified by polyols, the dispersion stabilities of the nanoparticles in water are quite different. MPEG-1200 molecular can provide much steric hindrance because of its inactive methyl terminal groups [27] and size effect, and with the static repulsion [2-4], to stabilize the dispersion of the nanoparticles in water. MPEG-coated MNPs can avoid forming network structure due to the inert

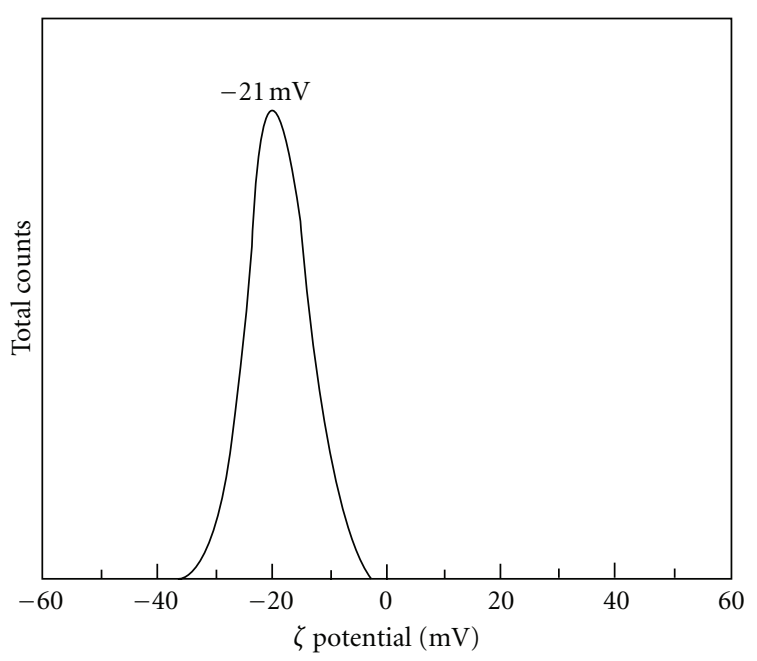

Figure 4: The zeta potential measurement of the magnetite nanoparticles modified by MPEG-1200 dispersed in deionized water.
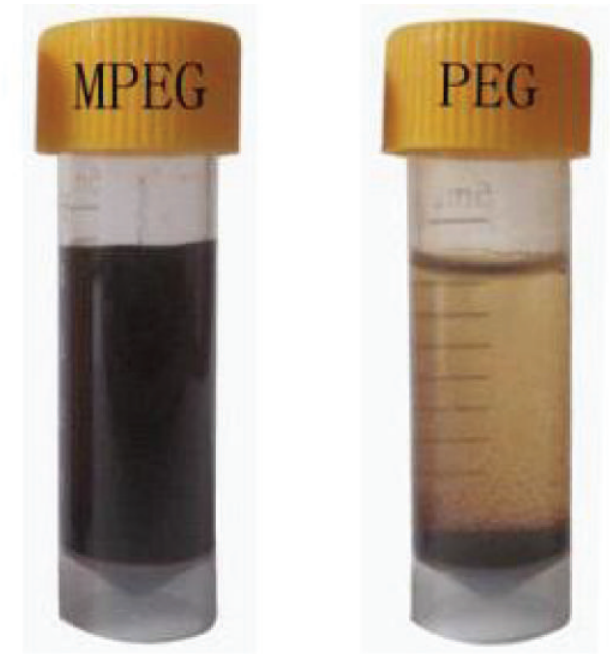

FIGURE 5: Colloidal stability tests of the magnetite nanoparticles modified by MPEG-1200 and by PEG-1000 in water.

terminal groups; however, PEG-coated MNPs form network structure easily (Scheme 1) [27].

The MPEG-coated MNPs were stable in water with small hydrodynamic size (Figure 6), which almost did not change for 3 months (Figure 7). This is an essential property that MNPs should have in order to meet the requirement in applications. On the contrary, the hydrodynamic size of the as-prepared MNPs modified by PEG-1000 was quite large (Figure 8), they precipitated in water for several days. This might be due to the possible formation of hydrogen bonding between adjacent PEGs coated on MNPs [25, 27]. It indicates that rather than the dissolution of PEGs from the surface of nanoparticles into water, which can cause aggregations of nanoparticles, the conjunction between surface molecular results in the poor water dispersion stability of the nanoparticles. 


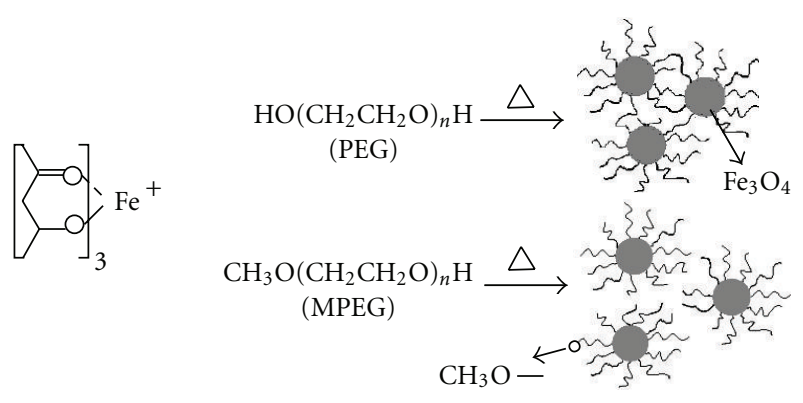

Scheme 1: Illustration of the magnetite nanoparticles synthesized by the thermal decomposition of $\mathrm{Fe}(\mathrm{acac})_{3}$ in PEG and in MPEG.

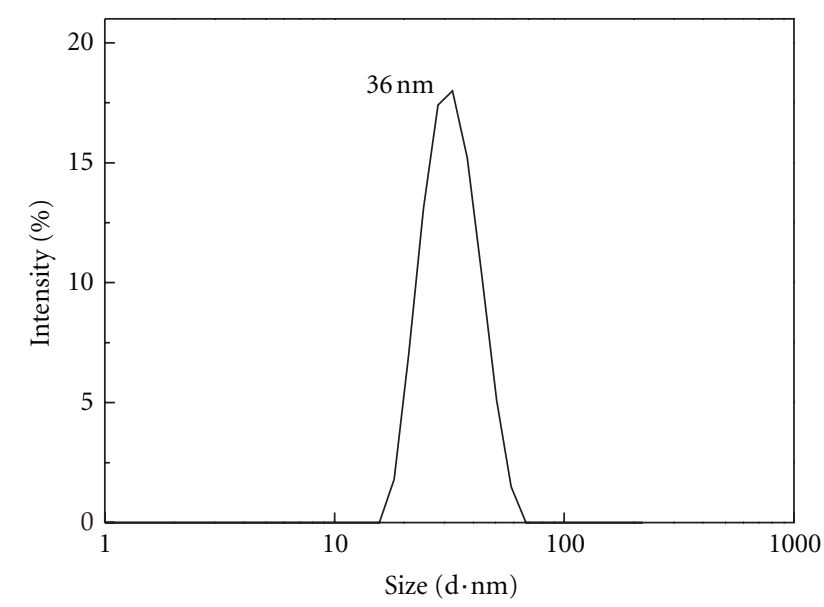

FIGURE 6: The hydrodynamic size of the magnetite nanoparticles synthesized by the thermal decomposition of Fe(acac $)_{3}$ in MPEG1200.

Figure 9 shows the hysteretic M-H curve of the MPEGcoated MNPs at $300 \mathrm{~K}$. Zero coercivity and zero remanence on the magnetization curves indicate superparamagnetic behavior of the MNPs, the saturation magnetization of the MNPs was $48 \mathrm{emu} / \mathrm{g}$. Thermogravimetric analysis of the MNPs synthesized in MPEG-1200 shows the presence of 19\% of polymer, so the saturation magnetization of $\mathrm{Fe}_{3} \mathrm{O}_{4}$ can be adjusted to $59 \mathrm{emu} / \mathrm{g}$.

Choosing MPEG as the modification agent is also based on the purpose that we expect the MNPs can be used as the fMRI contrast agents. Longer time circulation in blood and more stable concentration in blood over time are critical requirement in that application $[1,3,4,6]$. MPEG-coated MNPs that have no reactive $-\mathrm{OH}$ terminal group as compared to PEG-coated MNPs on their surfaces may meet the requirement.

\section{Conclusions}

The MNPs were synthesized by the thermal decomposition of $\mathrm{Fe}(\mathrm{acac})_{3}$ in MPEG-1200. The nanoparticles show superparamagnetic behavior in $300 \mathrm{~K}$. The negatively charged nanoparticles with small hydrodynamic size exhibited longer time dispersion in water than the MNPs synthesized by

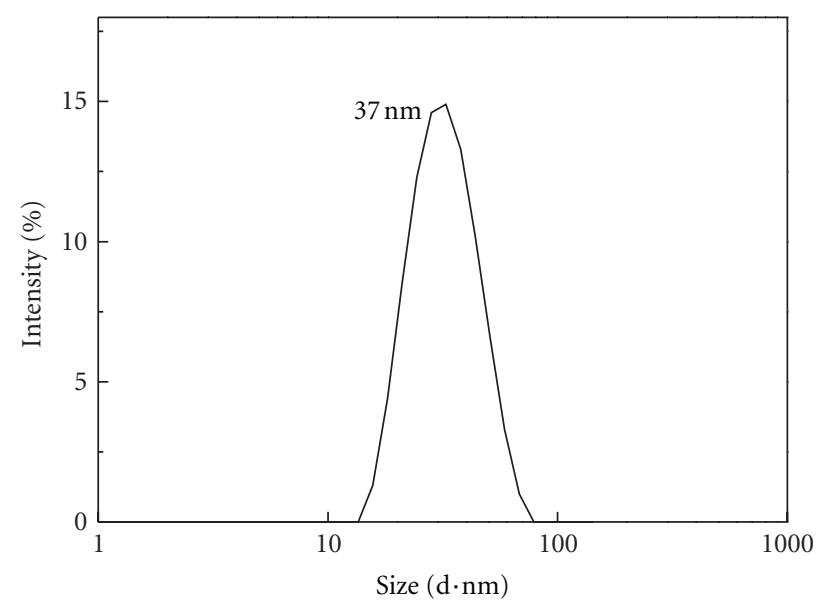

FIGURE 7: The hydrodynamic size of the magnetite nanoparticles synthesized by the thermal decomposition of $\mathrm{Fe}(\mathrm{acac})_{3}$ in MPEG1200 after being dispersed in water for 3 months.

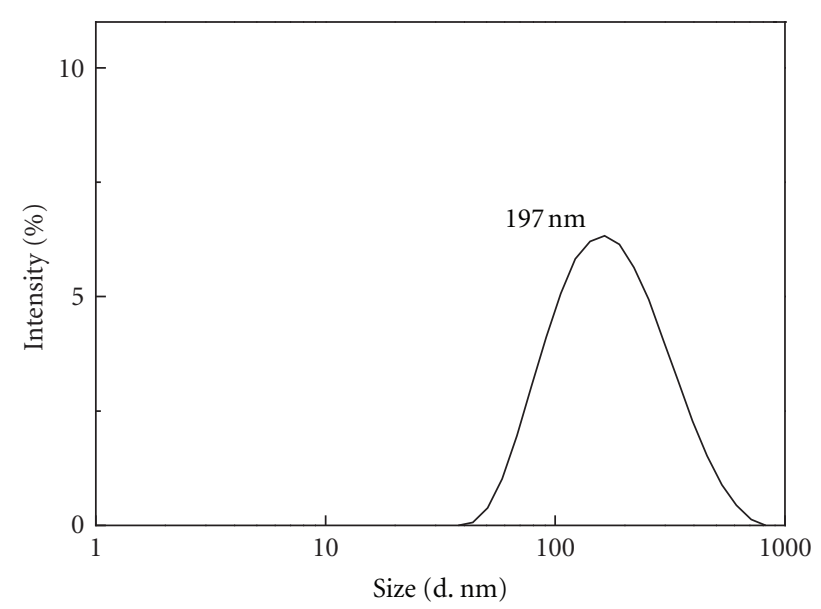

FIGURE 8: The hydrodynamic size of the magnetite nanoparticles synthesized by the thermal decomposition of $\mathrm{Fe}(\mathrm{acac})_{3}$ in PEG1000.

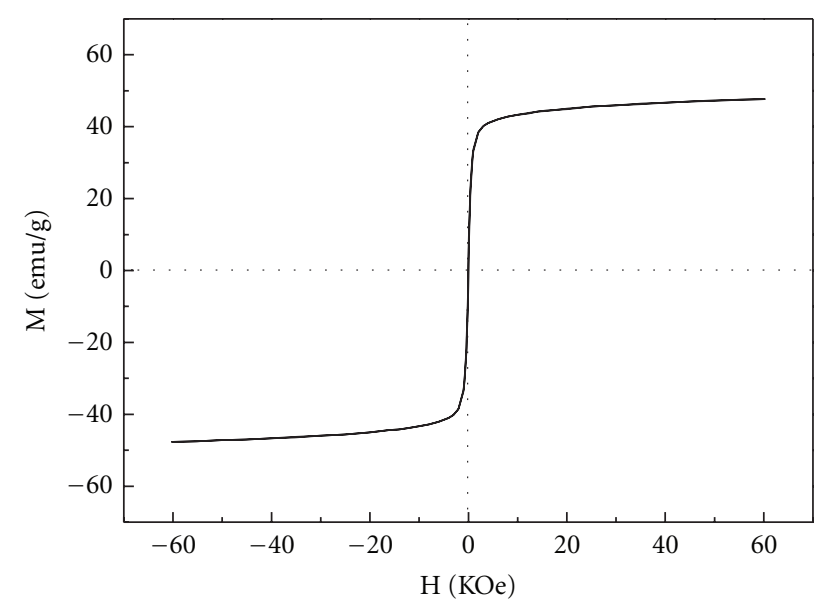

FIGURE 9: M-H curve of the magnetite nanoparticles (with the modification layer) synthesized by the thermal decomposition of $\mathrm{Fe}(\mathrm{acac})_{3}$ in MPEG-1200. 
the thermal decomposition of $\mathrm{Fe}(\mathrm{acac})_{3}$ in PEG-1000. The reduced conjunction between nanoparticles due to the inert $-\mathrm{CH}_{3}$ terminal group, the steric hindrance and the electrostatic repulsion forces between the MPEG-coated nanoparticles altogether could cause the higher stability in water dispersion. Future work can be done on the testing of the nanoparticles after treatments strictly according to medical procedures as contrast agents in magnetic resonance imaging such as MR angiography and cerebral blood pool imaging for fMRI.

\section{Acknowledgment}

This work was supported by the National Natural Science Foundation of China (no. 50962005 and no. 51162003).

\section{References}

[1] R. Weissleder, A. Bogdanov, E. A. Neuwelt, and M. Papisov, "Long-circulating iron oxides for MR imaging," Advanced Drug Delivery Reviews, vol. 16, no. 2-3, pp. 321-334, 1995.

[2] E. H. Kim, Y. Ahn, and H. S. Lee, "Biomedical applications of superparamagnetic iron oxide nanoparticles encapsulated within chitosan," Journal of Alloys and Compounds, vol. 434435, pp. 633-636, 2007.

[3] R. Qiao, C. Yang, and M. Gao, "Superparamagnetic iron oxide nanoparticles: from preparations to in vivo MRI applications," Journal of Materials Chemistry, vol. 19, no. 35, pp. 6274-6293, 2009.

[4] J. S. Weinstein, C. G. Varallyay, E. Dosa et al., "Superparamagnetic iron oxide nanoparticles: diagnostic magnetic resonance imaging and potential therapeutic applications in neurooncology and central nervous system inflammatory pathologies, a review," Journal of Cerebral Blood Flow and Metabolism, vol. 30, no. 1, pp. 15-35, 2010.

[5] B. Feng, R. Y. Hong, Y. J. Wu et al., "Synthesis of monodisperse magnetite nanoparticles via chitosan-poly(acrylic acid) template and their application in MRI," Journal of Alloys and Compounds, vol. 473, no. 1-2, pp. 356-362, 2009.

[6] C. Corot, P. Robert, J. M. Idée, and M. Port, "Recent advances in iron oxide nanocrystal technology for medical imaging," Advanced Drug Delivery Reviews, vol. 58, no. 14, pp. 14711504, 2006.

[7] K. Müller, J. N. Skepper, T. Y. Tang et al., "Atorvastatin and uptake of ultrasmall superparamagnetic iron oxide nanoparticles (Ferumoxtran-10) in human monocyte-macrophages: implications for magnetic resonance imaging," Biomaterials, vol. 29, no. 17, pp. 2656-2662, 2008.

[8] C. Fang and M. Zhang, "Multifunctional magnetic nanoparticles for medical imaging applications," Journal of Materials Chemistry, vol. 19, no. 35, pp. 6258-6266, 2009.

[9] M. A. McAteer, N. R. Sibson, C. von Zur Muhlen et al., "In vivo magnetic resonance imaging of acute brain inflammation using microparticles of iron oxide," Nature Medicine, vol. 13, no. 10 , pp. 1253-1258, 2007.

[10] A. Heymer, D. Haddad, M. Weber et al., "Iron oxide labelling of human mesenchymal stem cells in collagen hydrogels for articular cartilage repair," Biomaterials, vol. 29, no. 10, pp. 1473-1483, 2008.

[11] D. A. Orringer, Y. E. Koo, T. Chen, R. Kopelman, O. Sagher, and M. A. Philbert, "Small solutions for big problems: the application of nanoparticles to brain tumor diagnosis and therapy," Clinical Pharmacology and Therapeutics, vol. 85, no. 5, pp. 531-535, 2009.

[12] J. H. Park, G. Von Maltzahn, L. Zhang et al., "Magnetic iron oxide nanoworms for tumor targeting and imaging," Advanced Materials, vol. 20, no. 9, pp. 1630-1635, 2008.

[13] H. L. Liu, M. Y. Hua, H. W. Yang et al., "Magnetic resonance monitoring of focused ultrasound/magnetic nanoparticle targeting delivery of therapeutic agents to the brain," Proceedings of the National Academy of Sciences of the United States of America, vol. 107, no. 34, pp. 15205-15210, 2010.

[14] D. Shi, J. P. Cheng, F. Liu, and X. B. Zhang, "Controlling the size and size distribution of magnetite nanoparticles on carbon nanotubes," Journal of Alloys and Compounds, vol. 502, no. 2, pp. 365-370, 2010.

[15] Z. Xu, C. Shen, Y. Hou, H. Gao, and S. Sun, "Oleylamine as both reducing agent and stabilizer in a facile synthesis of magnetite nanoparticles," Chemistry of Materials, vol. 21, no. 9, pp. 1778-1780, 2009.

[16] R. Hao, R. Xing, Z. Xu, Y. Hou, S. Goo, and S. Sun, "Synthesis, functionalization, and biomedical applications of multifunctional magnetic nanoparticles," Advanced Materials, vol. 22, no. 25, pp. 2729-2742, 2010.

[17] J. Cheon, N. J. Kang, S. M. Lee, J. H. Lee, J. H. Yoon, and S. J. Oh, "Shape evolution of single-crystalline iron oxide nanocrystals," Journal of the American Chemical Society, vol. 126, no. 7, pp. 1950-1951, 2004.

[18] N. R. Jana, Y. Chen, and X. Peng, "Size- and shape-controlled magnetic ( $\mathrm{Cr}, \mathrm{Mn}, \mathrm{Fe}, \mathrm{Co}, \mathrm{Ni}$ ) oxide nanocrystals via a simple and general approach," Chemistry of Materials, vol. 16, no. 20, pp. 3931-3935, 2004.

[19] D. L. Zhao, X. W. Zeng, Q. S. Xia, and J. T. Tang, "Preparation and coercivity and saturation magnetization dependence of inductive heating property of $\mathrm{Fe}_{3} \mathrm{O}_{4}$ nanoparticles in an alternating current magnetic field for localized hyperthermia," Journal of Alloys and Compounds, vol. 469, no. 1-2, pp. 215218, 2009.

[20] Z. Li, L. Wei, M. Gao, and H. Lei, "One-pot reaction to synthesize biocompatible magnetite nanoparticles," Advanced Materials, vol. 17, no. 8, pp. 1001-1005, 2005.

[21] A. J. Cole, A. E. David, J. Wang, C. J. Galbán, H. L. Hill, and V. C. Yang, "Polyethylene glycol modified, cross-linked starchcoated iron oxide nanoparticles for enhanced magnetic tumor targeting," Biomaterials, vol. 32, no. 8, pp. 2183-2193, 2011.

[22] K. Hervé, L. Douziech-Eyrolles, E. Munnier et al., "The development of stable aqueous suspensions of PEGylated SPIONs for biomedical applications," Nanotechnology, vol. 19, no. 46, Article ID 465608, 7 pages, 2008.

[23] J. Wan, W. Cai, X. Meng, and E. Liu, "Monodisperse watersoluble magnetite nanoparticles prepared by polyol process for high-performance magnetic resonance imaging," Chemical Communications, no. 47, pp. 5004-5006, 2007.

[24] R. H. Gonalves, C. A. Cardoso, and E. R. Leite, "Synthesis of colloidal magnetite nanocrystals using high molecular weight solvent," Journal of Materials Chemistry, vol. 20, no. 6, pp. 1167-1172, 2010.

[25] Y. Xu, Y. Qin, S. Palchoudhury, and Y. Bao, "Water-soluble iron oxide nanoparticles with high stability and selective surface functionality," Langmuir, vol. 27, no. 14, pp. 8990-8997, 2011.

[26] N. Miguel-Sancho, O. Bomatí-Miguel, G. Colom, J. P. Salvador, M. P. Marco, and J. Santamaría, "Development of stable, water-dispersible, and biofunctionalizable superparamagnetic iron oxide nanoparticles," Chemistry of Materials, vol. 23, no. 11, pp. 2795-2802, 2011. 
[27] Q. J. Jia, J. F. Zeng, R. R. Qiao et al., "Gelification: an effective measure for achieving differently sized biocompatible $\mathrm{Fe}_{3} \mathrm{O}_{4}$ nanocrystals through a single preparation recipe," Journal of the American Chemical Society, vol. 133, no. 48, pp. 1951219523, 2011. 

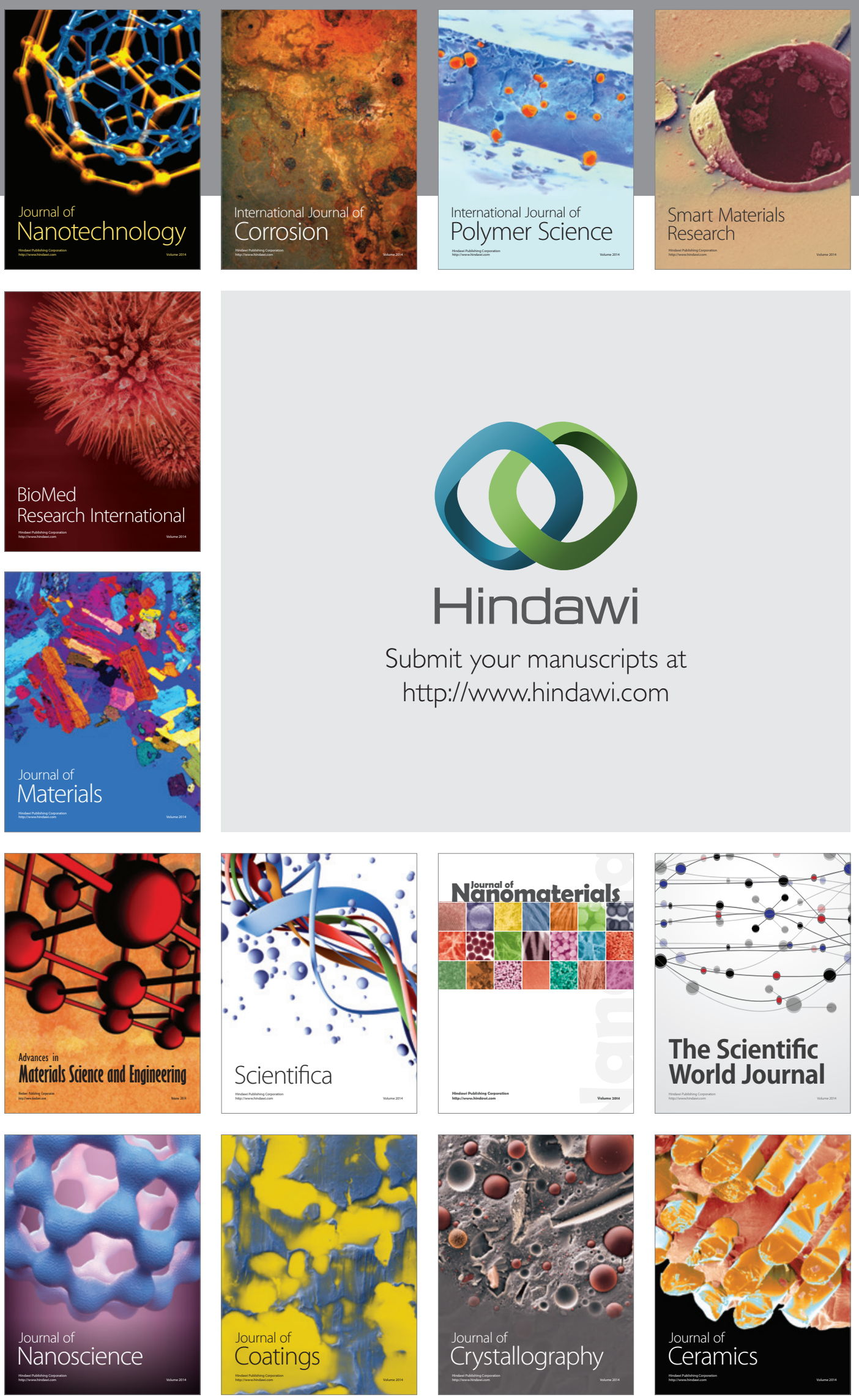

The Scientific World Journal

Submit your manuscripts at

http://www.hindawi.com

\section{World Journal}

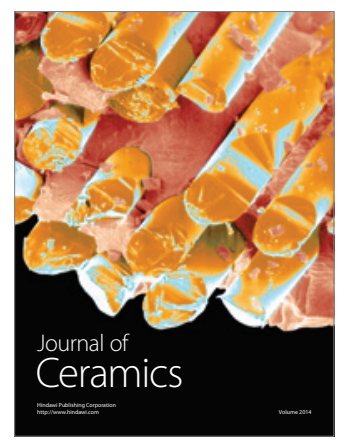

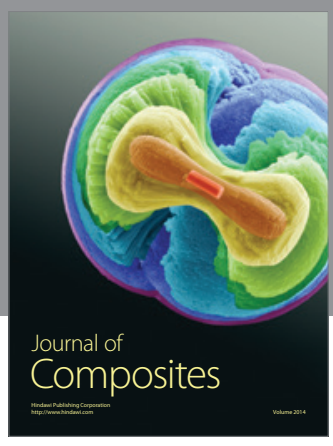
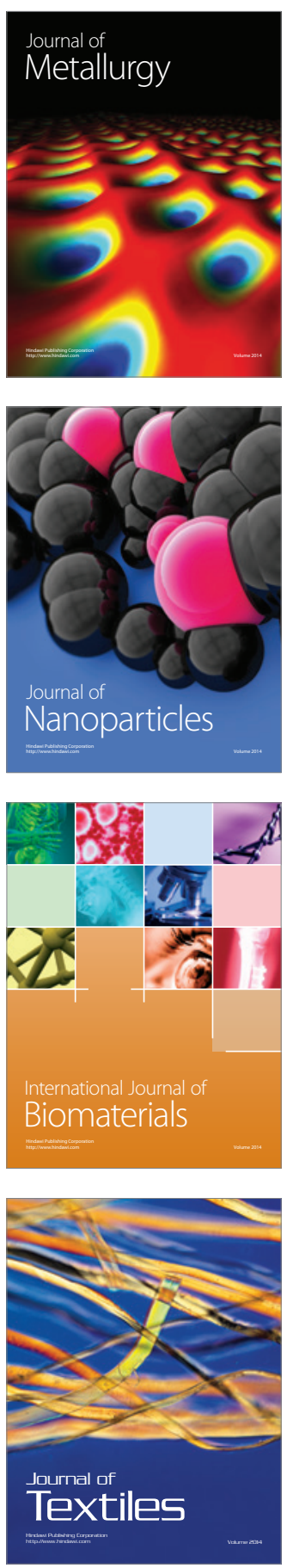